\title{
Uma análise acerca da humanização da assistência em unidades de terapia intensiva
}

\author{
An analysis about the humanization of assistance in intensive care units
}

Análisis sobre la humanización de la asistencia en unidades de cuidado intensivo

Rosana Serejo dos Santos ${ }^{1 *}$, Alanne Késsia de Souza Paiva ${ }^{1}$, Luciana Ribeiro de Carvalho ${ }^{1}$, Maria Tamires Alves Ferreira ${ }^{1}$, Bruna de Abreu Sepúlvedra Reis ${ }^{1}$, Adriana Rodrigues Alves de Sousa ${ }^{1}$, Solange Cristina Ferreira Queiroz ${ }^{1}$, Valdeane Silva Santos ${ }^{1}$, Lidiane Paiva Dias ${ }^{2}$, Nathália Rennê Damasceno e Silva Oliveira ${ }^{3}$.

\section{RESUMO}

Objetivo: Identificar as principais evidências científicas sobre a humanização do cuidado nas Unidades de Terapia Intensiva nos últimos cinco anos. Métodos: Trata-se de uma revisão integrativa realizada nas bases de dados Literatura Latino-Americana e do Caribe (LILACS) e Base de Dados de Enfermagem (BDENF) via Biblioteca Virtual em Saúde (BVS) usando os descritores em saúde (Decs) "assistência à saúde", "humanização da assistência" e "Unidades de Terapia Intensiva". Resultados: Foram identificados 11 artigos que compuseram a revisão. Que evidenciaram um dos principais objetivos do cuidado humanizado em Unidades de Terapia Intensiva relaciona-se à necessidade de manutenção da dignidade do ser humano e o respeito por seus direitos em todas as fases da vida. Mas, além disso, ele envolve também a forma de gerir os processos de trabalho em saúde para o alcance de melhorias, não só individuais, mas também coletivopessoais e estruturais nas instituições de saúde Considerações finais: Com a realização do presente estudo foi possível constatar que para a humanização assistencial nas Unidades de Terapia Intensiva é necessário aliar a utilização da tecnologia disponível à empatia, resinificando o que se compreende sobre cuidado, que dever ser entendido como um relacionamento interpessoal terapêutico seguro, responsável e ético a indivíduos.

Palavras-chave: Assistência à saúde, Humanização da assistência, Unidades de terapia intensiva.

\section{ABSTRACT}

Objective: Identify the main scientific evidence on the humanization of care in Intensive Care Units in the last five years. Methods: This is an integrative review carried out in the Latin American and Caribbean Literature (LILACS) and Nursing Database (BDENF) databases via the Virtual Health Library (VHL) using the health descriptors (Decs) " health care "," humanization of care "and" Intensive Care Units ". Results: 11 articles that comprised the review were identified. Which evidenced one of the main objectives of humanized care in Intensive Care Units is related to the need to maintain the dignity of human beings and respect for their rights at all stages of life. But, in addition, it also involves how to manage health work processes to achieve improvements, not only individual, but also collective / personal and structural in health institutions. Final considerations: With this study, it was possible to verify that for the assistance humanization in Intensive Care Units, it is necessary to combine the use of available technology with empathy, resinifying what is understood about care, which should be understood as a safe, responsible and ethical therapeutic interpersonal relationship to individuals.

Keywords: Health care, Humanization of care, Intensive care units.

\footnotetext{
${ }^{1}$ Faculdade Estácio de Teresina, Teresina - PI. *E-mail: enfer.rosanaserejo@gmail.com.

2 Universidade Federal do Piauí (UFPI), Teresina - PI.

${ }^{3}$ Faculdade Venda Nova do Imigrante (FAVENI), Teresina - PI.
}

SUBMETIDO EM: 9/2020

ACEITO EM: 10/2020

PUBLICADO EM: 12/2020 


\section{RESUMEN}

Objetivo: Identificar las principales evidencias científicas sobre la humanización de los cuidados en Unidades de Cuidados Intensivos en los últimos cinco años. Métodos: Se trata de una revisión integradora realizada en las bases de datos de Literatura Latinoamericana y del Caribe (LILACS) y Base de Datos de Enfermería (BDENF) a través de la Biblioteca Virtual en Salud (BVS) utilizando los descriptores de salud (Decs)". asistencia sanitaria "," humanización de la asistencia "y" Unidades de Terapia Intensiva ". Resultados: Se identificaron 11 artículos que componían la revisión. Lo que evidenció que uno de los principales objetivos de la atención humanizada en las Unidades de Cuidados Intensivos está relacionado con la necesidad de mantener la dignidad del ser humano y el respeto a sus derechos en todas las etapas de la vida. Pero, además, también involucra cómo gestionar los procesos de trabajo en salud para lograr mejoras, no solo individuales, sino también colectivas-personales y estructurales en las instituciones de salud. Consideraciones finales: Con este estudio fue posible constatar que para la humanización asistencial en las Unidades de Cuidados Intensivos es necesario combinar el uso de la tecnología disponible con la empatía, resinificando lo que se entiende sobre el cuidado, que debe entenderse como una relación interpersonal terapéutica segura, responsable y ética con las personas.

Palabras clave: Atención de salud, Humanización de la asistencia, Unidades de cuidados intensivos.

\section{INTRODUÇÃO}

A Unidade de Terapia Intensiva (UTI) foi idealizada como unidade de monitoração de paciente grave com base nas ações de Florence Nightingale realizadas em 1954, durante a guerra da Criméia. Os soldados feridos durante a guerra vinham a óbito pelas condições precárias, porém com as intervenções de cuidados baseado em grau de gravidade garantindo uma assistência mais complexas e especializada, onde os mais graves ficassem próximos à enfermagem com monitorização contínua, Florence obteve êxito com a redução da mortalidade (OUCHI JD, et al., 2018).

Assim nascia o objetivo básico da unidade de terapia intensiva de oferecer assistência avançada as funções vitais dos pacientes crítico ou semicrítico, mas que possuam chances de sobreviver ou que tenham o direito de morrer de forma digna e humana, em um ambiente físico e psicológico adequado (OUCHI JD, et al., 2018).

É um setor hospitalar que possui uma grande concentração de tecnologia, identificado como espaço destinado aos profissionais da saúde capacitados e especializados, com conhecimento e habilidade para a realização de procedimentos, sendo necessária interatividade e agilidade, uma vez que as decisões precisam ser rápidas e assertivas (FROTA LA, et al., 2016).

Dentre as finalidades das tecnologias em saúde estão a promover a saúde, prevenir e tratar doenças. Encontra-se divididas em: tecnologias duras referem-se ao uso de equipamentos tecnológicos, normas e estruturas organizacionais; tecnologias leveduras caracterizada pelos saberes que possibilita o processo de trabalho bem-sucedido e as tecnologias leves que se refere as tecnologias de relação e vínculo e acolhimento. Vale destacar que, discutir tecnologia não é discutir equipamento e nem o moderno e o novo, mas discutir o proceder eficaz de determinados saberes, procurando dessa forma, construir procedimentos de intervenção nos processos da saúde e da doença, do normal e do patológico, da vida e da morte, que produzam o efeito desejado (SANTOS WJ, et al., 2014).

Também se faz necessário práticas de atendimento às pacientes pautadas em um tratamento humanizado, demonstrando compreensão, atenção, gentileza e cortesia. Entende-se então, que humanização quer dizer riqueza em humanidade e compreende a essência do ser, o respeito à vida, a individualidade e a importância de um espaço concreto nas instituições da saúde que vise em primeiro lugar os seres humanos que estão envolvidos, abrangendo a visão do atendimento, que envolve prestar cuidados respeitando a individualidade do ser humano, aumentando a capacidade de entender a si mesmo e ao outro, ampliando a humanização de um conceito para uma ação (RADAELLI C, et al., 2019). 
Nesse cenário, a proposta de humanizar o trabalho em saúde surge no cenário das políticas públicas como uma oportunidade de propor, discutir e empreender um processo de mudança na cultura de atendimento vigente em toda a rede do Sistema Único de Saúde (SUS). Diante disso, com o objetivo de humanizar a assistência hospitalar prestada aos pacientes, o Ministério da Saúde criou em 2001, o Programa Nacional de Humanização da Assistência Hospitalar (PNHAH).

Esse programa é baseado em um processo de humanização dos serviços, de forma profunda e eficiente, processo esse destinado a provocar mudanças progressivas, sólidas e permanentes na cultura de atendimento à saúde, em benefício tanto dos usuários-clientes quanto dos profissionais (BRASIL, 2001).

Nesse contexto, destaca-se a relevância deste estudo, principalmente como uma contribuição críticoreflexiva sobre humanização no contexto da terapia intensiva, diante da importância em conhecer como os profissionais de saúde visualizam o cuidado em um ambiente altamente tecnológico. Assim, como o fato de que o conforto e a comunicação nas ações de cuidado ao paciente crítico ainda serem práticas distantes da realidade da maioria da população, transformando a UTI em um setor acompanhado por medos e incertezas dos usuários, familiares e trabalhadores de saúde. Diante do pressuposto, o presente estudo tem como objetivo identificar as principais evidências científicas sobre a humanização do cuidado nas Unidades de Terapia Intensiva nos últimos cinco anos.

\section{MÉTODOS}

Trata-se de estudo exploratório e descritivo, de abordagem ancorada em pesquisa bibliográfica do tipo revisão integrativa da literatura. Para elaboração deste estudo, necessitou-se percorrer seis etapas sistemáticas descritas por Mendes KDS, et al. (2019), seguindo deste a identificação do tema e escolha da questão norteadora; estabelecimento de critérios para inclusão e exclusão de estudos e busca na literatura; definição das informações a serem extraídas dos estudos; classificação dos estudos; avaliação e interpretação dos resultados; e por fim, a apresentação do estudo.

Primeiramente foi escolhida a temática a ser abordada e logo em seguida a formulação da questão norteadora por meio da estratégia PICo, um acrômio em que "P" é a população ou participantes (assistência à saúde), "l" o fenômeno de interesse (humanização) e "Co" para contexto do estudo (Unidades de Terapia Intensiva) (CANON M e BUITRAGO-GOMEZ Q, 2018). Dessa forma, definiu-se como questão norteadora do estudo "Quais as principais evidencias científicas acerca da humanização da assistência em Unidades de Terapia Intensiva nos últimos cinco anos?".

Após estabelecer as palavras chaves, para seguir na seleção foi necessário estabelecer os componentes estruturados, os descritores são estruturas hierárquicas que facilita a busca e recuperação de artigos e serve para organizar e promover fácil obtenção das informações coletadas. Optou-se pelos descritores em Ciências da Saúde (DeCS). Conforme descritos no Quadro 1.

Quadro 1 - Termos de busca utilizados no processo de investigação e seleção dos estudos primários.

\begin{tabular}{|c|c|c|c|}
\hline Descrição & Pico & Componentes & Descritores/ decs \\
\hline Participantes & P & Assistência à saúde & Assistência à saúde \\
\hline Interesse & $\mathrm{I}$ & Humanização & Humanização da Assistência \\
\hline Contexto & Co & $\begin{array}{c}\text { Unidades de Terapia } \\
\text { Intensiva }\end{array}$ & Unidades de Terapia Intensiva \\
\hline
\end{tabular}

Fonte: Santos RS, et al., 2020. 
Na segunda etapa foi realizada a busca nas bases de dados via Biblioteca Virtual em Saúde (BVS): Literatura Latino-Americana e do Caribe (LILACS) e Base de Dados de Enfermagem (BDENF). Foram definidos os DeCS "assistência à saúde", "humanização da assistência" e "Unidades de Terapia Intensiva"; associados entre si pelo operador boleano "AND". Conforme estratégia de busca descrita no Quadro 2.

Quadro 2 - Estratégia de busca dos artigos na base de dados.

\begin{tabular}{|c|c|}
\hline Base de dados & Estratégia de busca \\
\hline $\begin{array}{c}\text { LILACS e BDENF } \\
\text { VIA BVS }\end{array}$ & Tw:("assistência à saude" AND "humanização da assistencia" AND \\
& \begin{tabular}{c} 
"unidades de terapia intensiva") \\
\hline
\end{tabular} \\
\hline
\end{tabular}

Fonte: Santos RS, et al., 2020.

Foram selecionados somente os artigos publicados na integra, disponível gratuitamente em português, espanhol ou inglês e que tivessem sido publicados nos últimos 5 anos. Estabeleceram-se como critérios de exclusão, artigos duplicados, monografias ou teses, livros, resumos em eventos, revisões bibliográficas ou sistemáticas e estudos que não atendiam a questão norteadora e objetivo do estudo. Após a aplicação dos critérios de inclusão e exclusão foram incluídos no estudo 11 artigos.

Durante a terceira etapa as autoras construíram um quadro para extrair as informações, e facilitar a categorização das evidências a serem comparadas, com a finalidade de discutir os principais resultados e conclusões dos estudos.

Para análise do delineamento de pesquisa dos artigos e a avaliação quanto ao nível de evidência, optouse pela classificação e conceitos descritos por Polit DF e Beck CT (2019), para fundamentar esse estudo. Que avalia o nível de evidência quanto ao tipo de estudo, e varia de $1 \mathrm{~A}$ (revisões sistemáticas se Estudos controlados) a 7 (Opiniões de autoridades, comite de especialistas).

$\mathrm{Na}$ quarta etapa foi realizada uma análise criteriosa, procurando esclarecer os resultados diferentes encontrados nos diferentes estudos. Em seguida, na quinta etapa, voltou-se para a discussão dos principais achados, onde foi realizada uma avaliação crítica de cada artigo incluído na pesquisa, comparação entre os principais conteúdos abordados, identificando e estabelecendo as temáticas aborda nesta revisão com auxílio da Matriz de Amarração proposta por Mazzon JA (2018).

A Matriz de Amarração é reconhecido e amplamente utilizado no âmbito da administração de empresas que tem como objetivo analisar a aderência e compatibilidade ou associação de temática e objetivo (PEREIRA MCS, et al., 2020). Por fim, na última fase, buscou-se descrever de forma clara e sistemática todas as etapas percorridas pelos autores para a elaboração da pesquisa e apresentar os principais resultados evidenciados da análise dos artigos incluídos.

Inicialmente, identificou-se um total de 199 artigos nas bases via Biblioteca Virtual em Saúde (BVS), sendo 103 no LILACS e 96 na BDENF. Após aplicar os critérios de inclusão e exclusão, apenas 50 artigos permaneceram elegíveis para este estudo. Sendo que após leitura criteriosa dos títulos e objetivos, tiveram os resumos lidos na íntegra e passaram por uma leitura flutuante de todo o corpo do estudo visando identificar quais abordavam a temática pesquisada e que, desta forma, respondiam a questão norteadora do estudo. Ao final desta etapa se mantiveram no estudo apenas 11 , pois foram julgados coerentes com a proposta da revisão, como apresenta a Figura 1. 
Figura 1 - Fluxograma do caminho percorrido na seleção de artigos.

103 artigos foram excluídos por
apresentarem os seguintes critérios
de exclusão: monografias, livros,
tempo de publicação maior que 5
anos, não disponíveis na íntegra
(resumos em eventos) e revisão de
literature.

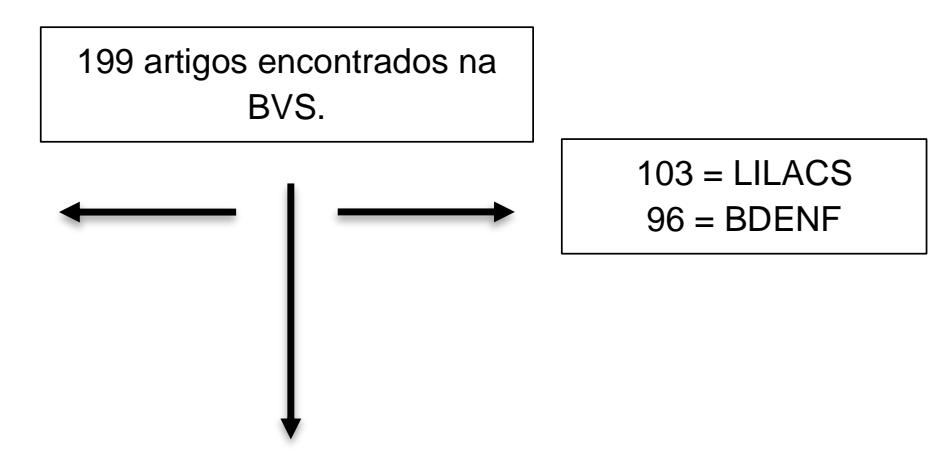
Resultando em 50artigos para análise criteriosa dos títulos e objetivos e duplicidade.

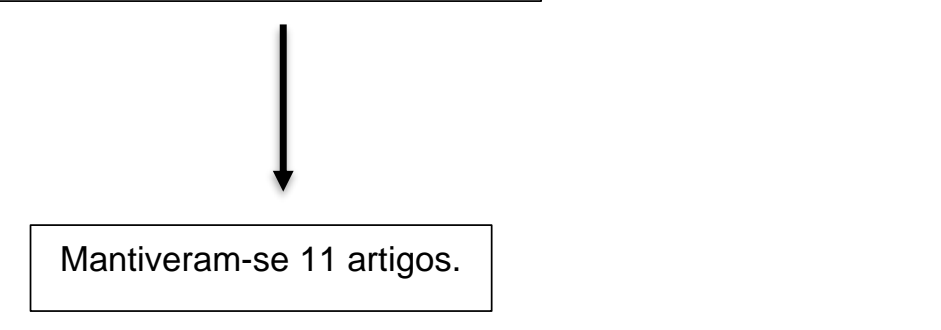

Fonte: SANTOS RS, et al., 2020.

Procedeu-se, então, a caracterização e o fichamento dos artigos, de modo a ordenar dentre outras variáveis: ano de publicação, base de dados, periódico, autor (citação), título, conclusão e nível de evidência cientifica. Em seguida, procedeu-se à avaliação e interpretação dos resultados, os quais foram sintetizados e organizados de modo a orientar a discussão e elaborar o documento final. Conforme demostrado no Quadro 3. 
Quadro 3 - Caracterização dos artigos selecionados para a análise de dados, segundo ano, base de dados, periódico, autor, título, objetivo, tipo de estudo e nível de evidência.

\begin{tabular}{|c|c|c|c|c|c|}
\hline $\begin{array}{l}\text { Ano/ } \\
\text { Base de } \\
\text { Dados }\end{array}$ & $\begin{array}{l}\text { Referência/ } \\
\text { Periódico }\end{array}$ & Título & Objetivo & Delineamento de Pesquisa & NE \\
\hline $\begin{array}{l}2018 \\
\text { BDENF }\end{array}$ & $\begin{array}{l}\text { (NODA LM, et } \\
\text { al., 2018) } \\
\text { REME rev. } \\
\text { min. Enferm }\end{array}$ & $\begin{array}{c}\text { A humanização em } \\
\text { Unidade de Terapia } \\
\text { intensiva neonatal sob a } \\
\text { ótica dos pais }\end{array}$ & $\begin{array}{c}\text { Compreender os significados de humanização } \\
\text { da assistência sob a ótica de pais de recém- } \\
\text { nascidos internados em uma Unidade de } \\
\text { Terapia Intensiva Neonatal }\end{array}$ & $\begin{array}{c}\text { Estudo descritivo e } \\
\text { Exploratório com abordagem } \\
\text { qualitativa }\end{array}$ & 6 \\
\hline $\begin{array}{l}2017 \\
\text { LILACS }\end{array}$ & $\begin{array}{l}\text { (VILLA LLO, } \\
\text { et al., 2017) } \\
\text { Rev. pesqui. } \\
\text { cuid fundam. } \\
\text { (Online); }\end{array}$ & $\begin{array}{l}\text { A percepção do } \\
\text { acompanhante sobre o } \\
\text { atendimento humanizado } \\
\text { em UTI pediátrica }\end{array}$ & $\begin{array}{c}\text { Compreender a percepção do acompanhante } \\
\text { da criança hospitalizada acerca do atendimento } \\
\text { humanizado no contexto da UTI pediátrica }\end{array}$ & $\begin{array}{c}\text { Estudo descritivo e } \\
\text { Exploratório com abordagem } \\
\text { qualitativa }\end{array}$ & 6 \\
\hline $\begin{array}{l}2017 \\
\text { LILACS / } \\
\text { BDENF }\end{array}$ & $\begin{array}{l}\text { (CARDOSO } \\
\text { OPJ, et al., } \\
\text { 2017) } \\
\text { Rev Rene } \\
\text { (Online) }\end{array}$ & $\begin{array}{l}\text { Com palavras não sei } \\
\text { dizer: resinificando o } \\
\text { cuidado através da } \\
\text { música em pós- } \\
\text { operatório } \\
\text { cardiopediátrico }\end{array}$ & $\begin{array}{c}\text { Compreender as ressignificações que a música } \\
\text { promove no cuidado às crianças em pós- } \\
\text { operatório cardíaco. }\end{array}$ & $\begin{array}{c}\text { Trata-se de uma pesquisa } \\
\text { qualitativa. }\end{array}$ & 6 \\
\hline $\begin{array}{l}2017 \\
\text { BDENF }\end{array}$ & $\begin{array}{l}\text { (NUNES } \\
\text { ECDA, et al., } \\
\text { 2017) } \\
\text { Rev. enferm. } \\
\text { UFPE online; }\end{array}$ & $\begin{array}{l}\text { O outro lado da terapia } \\
\text { intensiva - percepções } \\
\text { no pós alta }\end{array}$ & $\begin{array}{c}\text { Desvelar os aspectos positivos da internação } \\
\text { em UTI eleitos pelos indivíduos que vivenciam } \\
\text { talexperiência em estado de consciência } \\
\text { preservado. }\end{array}$ & $\begin{array}{c}\text { Estudo qualitativo, descritivo e } \\
\text { exploratório }\end{array}$ & 6 \\
\hline
\end{tabular}

REAS/EJCH | Vol.12(12) | e5117 | DOI: https://doi.org/10.25248/reas.e5117.2020 Página 6 de 14 
Revista Eletrônica Acervo Saúde / Electronic Journal Collection Health ISSN 2178-2091

\begin{tabular}{|c|c|c|c|c|c|}
\hline $\begin{array}{l}\text { Ano/ } \\
\text { Base de } \\
\text { Dados }\end{array}$ & $\begin{array}{l}\text { Referência/ } \\
\text { Periódico }\end{array}$ & Título & Objetivo & Delineamento De Pesquisa & NE \\
\hline $\begin{array}{l}2016 \\
\text { LILACS }\end{array}$ & $\begin{array}{l}\text { (MONDADORI } \\
\text { AG, et al., } \\
\text { 2016) } \\
\text { Fisioter. } \\
\text { Pesqui }\end{array}$ & $\begin{array}{l}\text { Humanização da } \\
\text { fisioterapia em Unidade } \\
\text { de Terapia Intensiva } \\
\text { Adulto: estudo } \\
\text { transversal }\end{array}$ & $\begin{array}{c}\text { Verificar se a assistência Fisioterapêutica em } \\
\text { unidade de terapia intensiva é realizada de } \\
\text { forma humanizada }\end{array}$ & $\begin{array}{l}\text { Trata-se de um estudo de corte } \\
\text { transversal }\end{array}$ & 6 \\
\hline $\begin{array}{l}2016 \\
\text { LILACS/ } \\
\text { BDENF }\end{array}$ & $\begin{array}{l}\text { (EVANGELIS } \\
\text { TA VC, et al., } \\
\text { 2016) } \\
\text { Rev. bras. } \\
\text { Enferm }\end{array}$ & $\begin{array}{l}\text { Equipe multiprofissional } \\
\text { de terapia intensiva: } \\
\text { humanização e } \\
\text { fragmentação do } \\
\text { processo de trabalho }\end{array}$ & $\begin{array}{c}\text { Compreender o significado do cuidado } \\
\text { humanizado em unidades de terapia intensiva } \\
\text { considerando a vivência da equipe } \\
\text { multiprofissional. }\end{array}$ & $\begin{array}{l}\text { Estudo qualitativo, descritivo e } \\
\text { exploratório. }\end{array}$ & 6 \\
\hline $\begin{array}{l}2016 \\
\text { LILACS }\end{array}$ & $\begin{array}{c}\text { (MACHADO } \\
\text { ER e } \\
\text { SOARES NV, } \\
\text { 2016) } \\
\text { Rev. enferm. } \\
\text { cent.-Oeste } \\
\text { min; }\end{array}$ & $\begin{array}{l}\text { Humanização em UTI: } \\
\text { sentidos e significados } \\
\text { sob a ótica da equipe de } \\
\text { saúde }\end{array}$ & $\begin{array}{l}\text { Identificar as concepções dos profissionais da } \\
\text { saúde sobre a humanização. }\end{array}$ & $\begin{array}{l}\text { Trata-se de um estudo descritivo } \\
\text { com } \\
\text { abordagem qualitativa }\end{array}$ & 6 \\
\hline $\begin{array}{l}2016 \\
\text { LILACS }\end{array}$ & $\begin{array}{c}\text { (RODRIGUES } \\
\text { AC e } \\
\text { CALEGARI T, } \\
\text { 2016) } \\
\text { REME rev. } \\
\text { min. enferm; }\end{array}$ & $\begin{array}{l}\text { Humanização da } \\
\text { assistência na unidade } \\
\text { de terapia intensiva } \\
\text { pediátrica: perspectiva da } \\
\text { equipe de enfermagem }\end{array}$ & $\begin{array}{c}\text { Analisar a visão da equipe de enfermagem } \\
\text { sobre a humanização da assistência às } \\
\text { crianças e famílias na Unidade de Terapia } \\
\text { Intensiva Pediátrica (UTIP) }\end{array}$ & $\begin{array}{l}\text { Trata-se de estudo descritivo, } \\
\text { transversal, com abordagem } \\
\text { quantitativa. }\end{array}$ & 6 \\
\hline
\end{tabular}


Revista Eletrônica Acervo Saúde / Electronic Journal Collection Health ISSN 2178-2091

\begin{tabular}{|c|c|c|c|c|c|}
\hline $\begin{array}{l}\text { Ano/ } \\
\text { Base de } \\
\text { Dados }\end{array}$ & $\begin{array}{l}\text { Referência/ } \\
\text { Periódico }\end{array}$ & Título & Objetivo & Delineamento de Pesquisa & NE \\
\hline $\begin{array}{l}2016 \\
\text { LILACS }\end{array}$ & $\begin{array}{l}\text { (SANCHES } \\
\text { RCN, et al., } \\
2016) \\
\text { Esc. Anna } \\
\text { Nery Rev. } \\
\text { Enferm; }\end{array}$ & $\begin{array}{l}\text { Percepções de } \\
\text { profissionais de saúde } \\
\text { sobre a humanização em } \\
\text { unidade de terapia } \\
\text { intensiva adulto }\end{array}$ & $\begin{array}{l}\text { Compreender a percepção dos profissionais de } \\
\text { saúde quanto ao cuidado humanizado em uma } \\
\text { Unidade de Terapia Intensiva Adulto (UTI) }\end{array}$ & $\begin{array}{l}\text { Trata-se de um estudo de natureza } \\
\text { qualitativa, descritiva, explicativa }\end{array}$ & 6 \\
\hline $\begin{array}{l}2015 \\
\text { LILACS }\end{array}$ & $\begin{array}{c}\text { (CAMPONOG } \\
\text { ARA S, et al., } \\
\text { 2015) } \\
\text { Rev. enferm. } \\
\text { Cent. Oeste } \\
\text { Min }\end{array}$ & $\begin{array}{l}\text { Percepções de pacientes } \\
\text { pós alta da unidade de } \\
\text { cuidados intensivos } \\
\text { sobre a hospitalização } \\
\text { nesse setor }\end{array}$ & $\begin{array}{c}\text { Conhecer as percepções dos pacientes em } \\
\text { período pós-alta de Unidades de Cuidados } \\
\text { Intensivos }\end{array}$ & $\begin{array}{c}\text { A pesquisa tem abordagem } \\
\text { qualitativa, descritivo-exploratória. }\end{array}$ & 6 \\
\hline $\begin{array}{l}2015 \\
\text { LILACS }\end{array}$ & $\begin{array}{l}\text { (ROSEIRO } \\
\text { CP e PAULA } \\
\text { KMP, 2015) } \\
\\
\text { Estud. psicol. } \\
\text { (Campinas) }\end{array}$ & $\begin{array}{l}\text { Concepções de } \\
\text { humanização de } \\
\text { profissionais em } \\
\text { Unidades de Terapia } \\
\text { Intensiva Neonatal }\end{array}$ & $\begin{array}{l}\text { Investigar a concepção de humanização e de } \\
\text { cuidado humanizado da equipe de profissionais } \\
\text { da UTIN, bem como o relato de suas práticas } \\
\text { de assistência ao RN }\end{array}$ & A pesquisa tem análise quantitativa & 6 \\
\hline
\end{tabular}

Fonte: Santos RS, et al., 2020. 


\section{RESULTADOS}

Os 11 estudos selecionados possuem o total de 42 autores e nenhum autor esteve presente em mais de um artigo. Dentre os estudos selecionados observa-se que abordagem qualitativa é descrita em $9(81,81 \%)$ artigos e apenas $2(18,18 \%)$ artigos apresentam com abordagem quantitativa. Com relação à população de estudo evidencia-se que o grupo focal se divide em: profissionais de saúde com 6 artigos (54,54 \%), 3 artigos $(27,27 \%)$ artigos possui o foco aos pacientes e 2 estudos (18,18\%) tem com objeto de estudo a família.

Acerca da área de formação profissional dos autores destaca-se a Enfermagem com 7 artigos (63,63\%) formados exclusivamente por autores da área, um artigo $(9,09 \%)$ realizado exclusivamente por fisioterapeutas, um artigo $(9,09 \%)$ por psicólogos e dois artigos $(18,18 \%)$ de caráter multiprofissional ( 1 médica, 1 psicóloga e 2 acadêmicas de enfermagem).

De acordo com o delineamento de pesquisa, os estudos da presente revisão foram classificados de acordo com o nível de evidência adotado por Polit DF e Beck CT (2019). Onde todos os artigos (100\%) foram classificados com nível de 6 por ser estudos definidos principalmente como descritivos, esse baixo nível de evidência está relacionado ao delineamento não experimental adotado pelos autores incluídos na presente revisão.

Mesmo fracas as evidências dos estudos descritivos, a prática profissional depende de pesquisas descritivas ou experimentais que costuma apresentar uma progressão lógica de expansão do conhecimento, por isso, as mesmas não podem ser descartadas (POLIT DF e BECK CT, 2019).

Com o proposito de alinhar os artigos selecionados entre as temáticas e abordagens metodológicas, bem como auxiliar as pesquisadoras no planejamento e elaboração dos resultados deste estudo. Foi construída uma matriz, denominada Matriz de Amarração, proposta por Mazzon JA (2018), aqui adaptada, que mostrou ser uma ferramenta substancial para organizar a analise de conteúdo. A matriz elaborada para este estudo pode ser conferida no Quadro 4. 
Quadro 4 - Matriz de Amarração para relacionar os artigos entre si.

\begin{tabular}{|c|c|c|c|}
\hline Autores & $\begin{array}{l}\text { Quantidade de } \\
\text { Participantes } \\
\text { no estudo }\end{array}$ & $\begin{array}{l}\text { Técnica de } \\
\text { Estudo }\end{array}$ & $\begin{array}{c}\text { Temática na construção dos resultados e } \\
\text { discussão }\end{array}$ \\
\hline $\begin{array}{l}\text { (NODA LM, et al., } \\
\text { 2018) }\end{array}$ & $\begin{array}{l}14 \text { Pais com } \\
\text { filhos na UTIN }\end{array}$ & $\begin{array}{c}\text { Entrevista } \\
\text { semiestruturada }\end{array}$ & $\begin{array}{l}\text { Cuidados; Relacionamento família e } \\
\text { profissionais; } \\
\text { Comportamento dos profissionais }\end{array}$ \\
\hline $\begin{array}{l}\text { (VILLA LLO, et al., } \\
\text { 2017) }\end{array}$ & Familiares & $\begin{array}{c}\text { Entrevista } \\
\text { semiestruturada }\end{array}$ & $\begin{array}{l}\text { O cuidado humanizado; } \\
\text { A percepção do sofrimento }\end{array}$ \\
\hline $\begin{array}{l}\text { (CARDOSO OPJ, } \\
\text { et al., 2017) }\end{array}$ & 10 profissionais & Pesquisa ação & $\begin{array}{l}\text { A dinâmica de uma Unidade de Terapia } \\
\text { Intensiva Pediátrica }\end{array}$ \\
\hline $\begin{array}{l}\text { (NUNES ECDA, et } \\
\text { al., 2017) } \\
\end{array}$ & 11 pacientes & $\begin{array}{c}\text { Entrevista } \\
\text { semiestruturada }\end{array}$ & Apresentar o significado da experiência na UTI \\
\hline $\begin{array}{l}\text { (MONDADORI AG, } \\
\text { et al, 2016) }\end{array}$ & 60 Pacientes & $\begin{array}{l}\text { Entrevista face a } \\
\quad \text { face }\end{array}$ & $\begin{array}{l}\text { Satisfação do paciente com os procedimentos } \\
\text { de fisioterapia no momento da alta hospitalar. }\end{array}$ \\
\hline $\begin{array}{l}\text { (EVANGELISTA } \\
\text { VC, et al., 2016) }\end{array}$ & 24 Profissionais & $\begin{array}{c}\text { Entrevista } \\
\text { semiestruturada }\end{array}$ & As vertentes do cuidado prestado \\
\hline $\begin{array}{l}\text { (MACHADO ER e } \\
\text { SOARES NV } \\
\text { 2016) }\end{array}$ & 23 Profissionais & $\begin{array}{l}\text { Questionário } \\
\text { Estruturado }\end{array}$ & $\begin{array}{l}\text { O (des) conhecimento (PNH) pela equipe da } \\
\text { UTI; Humanização em UTI: múltiplas } \\
\text { dimensões do cuidado ao paciente, família e } \\
\text { equipe; Estratégias para Humanização no } \\
\text { cenário da UTI. }\end{array}$ \\
\hline $\begin{array}{l}\text { (RODRIGUES AC } \\
\text { e CALEGARI T, } \\
\text { 2016) }\end{array}$ & $\begin{array}{l}28 \text { profissionais } \\
\text { da equipe de } \\
\text { enfermagem }\end{array}$ & $\begin{array}{l}\text { Questionário } \\
\text { estruturado }\end{array}$ & $\begin{array}{l}\text { Reflexão acerca de sua assistência, sendo } \\
\text { possível o repensar da postura profissional } \\
\text { diante da prática do cuidado, buscando torná- } \\
\text { lo acolhedor e humanizado }\end{array}$ \\
\hline $\begin{array}{l}\text { (SANCHES RCN, } \\
\text { et al, 2016) }\end{array}$ & 13 profissionais & $\begin{array}{c}\text { Entrevista } \\
\text { semiestruturadas }\end{array}$ & $\begin{array}{l}\text { A apresentação dos resultados, por meio da } \\
\text { composição de duas categorias gerais: } \\
\text { conceito de humanização e as dificuldades } \\
\text { encontradas pelos profissionais para a } \\
\text { aplicabilidade e efetividade da humanização } \\
\text { do cuidado. }\end{array}$ \\
\hline $\begin{array}{l}\text { (CAMPONOGARA } \\
\text { S, et al., 2015) }\end{array}$ & $\begin{array}{c}14 \text { Pacientes } \\
\text { (saturação dos } \\
\text { dados) }\end{array}$ & $\begin{array}{c}\text { Entrevista } \\
\text { semiestruturada }\end{array}$ & $\begin{array}{l}\text { Experiência da hospitalização; } \\
\text { A religiosidade como estratégia de } \\
\text { enfrentamento. }\end{array}$ \\
\hline $\begin{array}{l}\text { (ROSEIRO CP e } \\
\text { PAULA KMP } \\
\text { 2015) }\end{array}$ & $\begin{array}{l}29 \text { profissionais } \\
\text { de saúde } \\
\text { atuantes na } \\
\text { UTIN }\end{array}$ & $\begin{array}{c}\text { Entrevista } \\
\text { semiestruturada }\end{array}$ & Uma análise situacional da assistência \\
\hline
\end{tabular}

Legenda: UTI: Unidades de Terapia Intensiva; UTIN: Unidades de Terapia Intensiva Neonatal; PNH: Politica Nacional de Humanização (PNH).

Fonte: Santos RS, et al., 2020, adaptado de Mazzon JA (2018). 
Após avaliar as características dos artigos com relação às temáticas abordadas, população do estudo, aplicação da metodologia, resultados e os principais temas da discussão, conforme apresentada na Matriz de Amarração, e análise dos estudos emergiram três categorias pertinentes que revelam o que a produção científica traz sobre a temática, são elas: Polica Nacional Humanização: Conceitos e cuidado humanizado; Relação pacientes, familiares e profissionais; e Assistência humanizada em UTIs e desafios.

\section{DISCUSSÃO}

\section{Politica nacional de humanização: conceitos e cuidado humanizado.}

As políticas de humanização foram implantadas com o objetivo de melhorar a assistência prestada pelos serviços de saúde. Assim surgiram a Política de Humanização da Assistência Hospitalar, o Programa Mãe Canguru e o Hospital Amigo da Criança. Esses avanços vêm se intensificando devido aos esforços conjuntos do Ministério da Saúde e, principalmente, da sociedade (GIACOMELLO KJ e MELO LL, 2019).

Machado ER e Soares NV (2016) constaram em seus estudos que os profissionais atuantes na UTI, apesar da maioria não ter conhecimento do conteúdo da Política Nacional de Humanização (PNH) na íntegra, trazem para sua prática diária valores como respeito, dignidade e amor ao próximo, tentando assim tornar mais humanas as suas atividades diárias. Contudo, a implantação de programas de humanização do cuidado em ambiente hospitalar parece ainda não ser uma realidade em todas as instituições de saúde, necessitando de maior discussão e empenho dos gestores e profissionais visando à concretização dessa política nesse cenário.

Para Sanches RCN, et al. (2016), os profissionais de saúde possuem algum conhecimento acerca do tema, em concordância aos direcionamentos previstos pela $\mathrm{PNH}$, porém de maneira dispersa. Tal percepção sugere certa incoerência entre o cuidado humanizado, preconizado pela $\mathrm{PNH}$, e a prática assistencial, configurando a existência das práxis no cotidiano de trabalho. Atribui-se essa problemática à falta de uma reflexão mais ampla sobre a humanização em saúde, desde a formação profissional, buscando valorizar o processo de desenvolvimento de competências ético-morais que orientem a futura prática.

\section{Relação paciente, familiares e profissionais. .}

Em relação à família, sua inserção é muito mencionada nos estudos como parte integrante do processo de humanização. Os artigos trazem que o envolvimento da equipe de saúde com as famílias dos pacientes é um importante pré-requisito para a humanização, visto que quando há uma boa relação entre profissionais e familiares, facilita-se o processo de participação familiar no tratamento e consequente recuperação do paciente.

Para Roseiro CP e Paula KMP (2015), os profissionais entendem a humanização a partir de uma representação da relação paciente-profissional, por meio de uma assistência centrada no respeito à pessoa humana, em uma melhor comunicação e orientação ao paciente e sua família, e, sobretudo, em seu acolhimento. Considerando a equipe como elo fundamental no processo de cuidado.

Em seu estudo, Noda LM, et al. (2018) buscaram compreender o conhecimento da familia, em especial os pais, sobre a humanização durante o processo assistencial prestado aos recém-nascidos nas Unidades de Terapia Intensiva Neonatal (UTIN) para auxiliar na elaboração e planejamento de estrategias para inserir a familia no processo de internação do recem-nascido $(R N)$ e garantir uma assistência de qualidade tanto ao paciente quanto para a familia.

Em relação à participação da família como parte integrante do processo de humanização destaca-se que o processo de crescimento e desenvolvimento da criança e o vínculo mãe-filho e pai-filho precisa ser mantido e estimulado, afinal os sentimentos e expectativas geradas no final da gestação de contato e ir pra casa com o novo membro da familia é interrompidos e esse recem-nascido passa a necissitar de cuidados intensivos e constantes, e apartir desse momento o ambiente passa ser cercado por medo, insegurança, normas e protocolos desconhecidos da familia (NODA LM, et al., 2018). 
Os pais consideram o cuidado humanizado como tanto pela maneira que se cuida e quanto pela relação que se estabelece com os profissionais de saúde. O comportamento do profissional, a comunicação efetiva, os cuidados individualizados ao RN e à família são premissas que devem ser levados em consideração pela equipe de saúde, que busca oferecer um cuidado humanizado (NODA LM, et al., 2018).

Villa LLO, et al. (2017) mostram o olhar dos acompanhantes e familiares, sobre o cuidado e acolhimento dos pacientes e a importância da atuação humanizada prestada pelos profissionais, e apontam esta humanização como responsável pela melhor compreensão do cuidado nas unidades de terapia intensiva, ganhando espaço como um local que possibilita a recuperação dos pacientes e não como um lugar destinado necessariamente a pacientes sem chances de sobrevivência.

Observa-se que a percepção dos acompanhantes sobre o conceito de humanização envolve atenção, zelo, preocupação e muitas vezes até um envolvimento afetivo com o paciente. Revelando em seus depoimentos que apesar de se tratar de uma UTI, onde o estresse do trabalho é grande, os profissionais envolvidos na assistência atuam de forma humanizada, com carinho, respeito e compreensão com a criança, possibilitando, uma aliança entre os avanços tecnológicos existentes e uma assistência mais humana (VILLA LLO, et al., 2017).

Nesse sentido, para Camponogara S, et al. (2015), qualquer atividade de assistência protagonizada pelos diversos profissionais da área da saúde, em diferentes cenários, deve ter a humanização como mola mestra. Nos ambientes de terapia intensiva, dadas as suas características peculiares, acredita-se que seja ainda mais relevante orientar o cuidado à saúde com base nesse conceito, torna-se fundamental conhecer a percepção dos sujeitos que vivenciam esse processo.

Dentre esses diversos profissionais, no estudo de Mondadori et al (2016) identifica-se os fisioterateutas. Nele, os autores concluíram que os procedimentos de fisioterapia são muitas vezes invasivos e causadores de desconforto e estresse para os pacientes, à vista disso, os profissionais devem realizá-los com atitudes humanizadas, e buscando, além da cura física, a cura emocional desses pacientes, trabalhando com ética pela dignidade humana, atendendo a satisfação dos pacientes.

Corroborando com Mondadori AG, et al. (2016), a pesquisa de Camponogara S, et al. (2015) aponta que a importância de uma atuação humanizada por parte da equipe multiprofissional, oferecendo ínclusive suporte emocional, no intuito de contribuir para minimizar a experiência negativa que cerceia a internação nesses setores. Que ao realizar os procedimentos que são necessarios nesse setor, que se utilize de empatia e comunicação, mesmo que o paciente naquele momento não esteja responsivo, deve informar as etapas e para que servem os procedimentos que irá realizar.

\section{Assistência humanizada em UTIs e desafios.}

A assistência humanizada deve envolver, além do paciente, seu contexto familiar e social, bem como a própria UTI e a equipe de saúde, ações associadas à valorização e respeito ao paciente crítico. Rodrigues AC e Calegari T (2016) aborda que a humanização deve transcender o enfoque no usuário e atentar para as circunstâncias favoráveis de trabalho e satisfação profissional, o que reflete na qualidade do atendimento.

Ainda de acordo com Rodrigues AC e Calegari T (2016), devem-se criar estratégias de compartilhamento de ideias e sentimentos, como em espaços para encontro entre profissionais e gestores, visando estabelecer uma efetiva assistência humanizada. Que a humanização seja baseada em um cuidado e um toque com diferencial, também para aos profissionais de saúde, à estrutura física do setor e à organização e gestão das instituições hospitalares.

Entender a humanização em UTI significa compreender como os aspectos organizacionais do trabalho neste setor influenciam no desenvovimento de atividades dos profissionais envolvidos. As tecnologias utilizadas para a realização do cuidado são de extrema importância para a reabilitação do indivíduo hospitalizado e requerem apropriação por parte dos profissionais que as utilizam. Contudo, deve ser valorizada a subjetividade de cada doente, sendo aspecto que pode dificultar a integralidade do cuidar (SANCHES RCN, et al., 2016). 
Vale destacar a importância do trabalho em equipe multiprofissional para promoção humanização, pois representa uma maneira dos profissionais atingirem a prática do cuidado humanizado por meio do fator "interação", que passa a ser inserido na organização do trabalho em saúde, e traz à tona a comunicação como um elo. Essa interação é apontada também junto à PNH como um fator importante dentro de horizonte normativo que guia as práticas de cuidado humanizado (EVANGELISTA VC, et al, 2016).

Cardoso OPJ, et al. (2017) apresentam a musicoterapia como intervenção na promoção da humanização da assistência. Concluíram que as dinâmicas e atribuições profissionais na assistência hospitalar em uma unidade pediátrica dificultam momentos de relaxamento e que essa tensão é percebida pelos pacientes e familiares. Entretanto, evidenciou-se que a música, enquanto complementação do cuidado ameniza sofrimentos e integra crianças a um lugar que, para elas, é inseguro e desconhecido, bem como cativa, envolve e emociona desde os pequenos aos profissionais. :

Nunes ECDA, et al. (2017) buscaram conhecer possíveis transformações na compreensão do indivíduo sobre a UTI antes e depois do seu internamento conseguiram constatar que o processo de humanização nas UTIs tem influenciado na desconstrução da visão estereotipada de tal ambiente.

Para a internação positiva é necessário que haja um comportamento de cuidado além dos aspectos técnicos, atentando-se ao paciente não somente para o seu contexto biológico, dando ênfase à integralidade do sujeito e compartilhando com ele seus sentimentos por meio do respeito e afetividade, recorrendo ao diálogo.

\section{CONSIDERAÇÕES FINAIS}

A presente revisão integrativa identificou as principais evidências científicas sobre a humanização do cuidado nas Unidades de Terapia Intensiva. Permitindo o aprofundamento teórico sobre a Prática baseada em humanização. Assim como identificou a necessidade de se desenvolverem estudos quase experimentais ou experimentais envolvendo a humanização, visto que todos os estudos desta revisão foram classificados com baixo nível de evidência. Apresentando a necessidade de estudos e pesquisas científicas com delineamentos que produzam evidências fortes, relacionadas ao tema é de grande relevância, visto que podem revelar como os profissionais se percebem diante da política e prática em saúde, bem como clarificar os princípios, métodos e diretrizes que fundamentam a PNH além de aumentar o poder de dos achados. Pretende-se que os resultados da presente revisão contribuam para construção do conhecimento da pratica profissional, mas que especialmente isto se traduza em melhoria dos cuidados, bem como estimular aos profissionais de saúde a se tornarem consumidores de resultados de pesquisa e especialmente serem produtores de pesquisas que possam ser utilizadas na prática clínica.

\section{REFERÊNCIAS}

1. BRASIL. Ministério da Saúde. Secretaria de Assistência à Saúde. Programa Nacional de Humanização da Assistência Hospitalar. Brasília (DF): 2001.

2. CAMARGO FC, et al. Modelos Para A Implementação Da Prática Baseada Em Evidências Na Enfermagem Hospitalar: Revisão Narrativa. Tex. Contex. - Enferm., 2017; 26(4): e2070017.

3. CAMPONOGARA $S$, et al. Percepções de pacientes pós-alta da unidade de cuidados intensivos sobre a hospitalização nesse setor. Rev. Enferm. Centr. Oes. Min., 2015; 5(1).

4. CANON M, BUITRAGO-GOMEZ Q. La pregunta de investigación en la práctica clínica: guía para formularla. Rev. Colomb. Psiquiatr., 2018; 47(3): 193-200.

5. CARDOSO OPJ, et al. Com palavras não sei dizer: Ressignificando o cuidado através da música em pós-operatório Cardiopediátrico. Rev Rene [Internet]. 2017;18(5):655-662.

6. EVANGELISTA VC, et al. Equipe multiprofissional de terapia intensiva: humanização e fragmentação do processo de trabalho. Rev. Bras. Enferm. 2016; 69(6): 1099-1107.

7. FROTA L A, et al. A visibilidade do enfermeiro em unidades de terapia intensiva: percepções de trabalhadores. Rev. Eletr. Enferm. 2016; 17(3).

8. GIACOMELLO KJ, MELO LL. O sentido do cuidado à criança hospitalizada: vivências de profissionais de enfermagem. Rev. Bras. Enferm., 2019; 72(3): 251-258.

9. MACHADO ER, SOARES NV. Humanização em UTI: Sentidos e Significados Sob a Ótica da Equipe de Saúde. Rev. Enferm. Cent. Oes. Min., 2016; 6(3). 
10. MAZZON JA. Using the Methodological Association Matrix in Marketing Studies. Rev. Bras. Marke., 2018; 17(5): 747770.

11. MENDES KDS, et al. Use of The Bibliographic Reference Manager In The Selection Of Primary Studies In Integrative Reviews. Tex. Contex. - Enferm, 2019; 28: e20170204.

12. MONDADORI AG, et al. Humanização da fisioterapia em Unidade de Terapia Intensiva Adulto: estudo transversal. Fisioter. Pesqui., 2016; 23(3): 294-300.

13. NODA LM, et al. Humanization in the Neonatal Intensive Care Unit from parents' perspective. REME: Rev. Min. Enferm., 2018; 22.

14. NUNES ECDA, et al. O outro lado da terapia intensiva - percepções no pós alta. Rev. Enferm. UFPE online, $2017 ; \mathrm{p}$. 5252-5258.

15. OUCHI JD, et al. O Papel do Enfermeiro na Unidade de Terapia Intensiva Diante de Novas Tecnologias em Saúde. Rev. Saú. em Foc. online. 2018; 10.

16. PEREIRA MCS, et al. Educação Ambiental e política pública educacional: uma abordagem interdisciplinar na perspectiva da intervenção social a partir do projeto Ocupe a Praça (São Paulo, SP). Revista Brasileira de Educação Ambiental (RevBEA), 2020; 15(1): p. 209-244.

17. POLIT DF, BECK CT. Fundamentos de pesquisa em enfermagem: avaliação de evidências para a prática da enfermagem. 9. ed. Porto Alegre: Artmed, 2019.

18. RADAELLI C, et al. O cuidado humanizado no ambiente de urgência e emergência: Uma revisão integrativa. Research, Society and Development, 2019; 8(6): 37861057.

19. RODRIGUES AC, CALEGARI T. Assistance Humanization in Pediatric Intensive Care Unit: Perspective Of Nursing Staff. REME: Rev. Min. Enferm. 2016; 20(0).

20. ROSEIRO CP, PAULA KMP. Concepções De Humanização De Profissionais Em Unidades De Terapia Intensiva Neonatal. Estud. psicol. (Campinas), 2015; 32(1): 109-119.

21. SANCHES RCN, et al. Perceptions of health professionals about humanization in intensive care unit adult. Escola Anna Nery - Rev. de Enferm. 2016; 20(1).

22. SANTOS WJ, et al. A. Avaliação da tecnologia das relações de cuidado nos serviços em saúde: percepção dos idosos inseridos na Estratégia Saúde da Família em Bambuí, Brasil. Ciênc. saúde coletiva, 2014; 19(8): 3441-345.

23. VILLA LLO, et al. A percepção do acompanhante sobre o atendimento humanizado em unidade de terapia intensiva pediátrica The perception of the companion of the humanized care in a pediatric intensive care unit. Rev de Pesq.: Cuid é Fund Online, 2017; 9(1): 187-19. 